\title{
STRATEGIES FOR ANESTHESIA MANAGEMENT OF CESAREAN DELIVERIES WITH ABNORMAL PLACENTAL PLACEMENT-ANESTHETIST'S PERSPECTIVE
}

\author{
Maqsood Ahmad \\ Combined Military Hospital Okara/National University of Medical Sciences (NUMS) Pakistan
}

ABSTRACT

\begin{abstract}
Objective: To discuss problems faced in the management of emergency and elective cesarean sections with known or unknown abnormal placental placement and their management.

Study Design: Retrospective observational study.

Place and Duration of Study: Different Hospitals for the last 7 years from 2013 to 2020.

Methodology: This retrospective observational study of 120 cases was selected for data collections that underwent anesthesia. Variables under study were age, weight, type of abnormal placenta, fluid, blood/blood products given during surgery, hysterectomy done, general surgeon help required, anesthesia type, and redo of operations.

Results: A total of 120 patients were operated having abnormal placentation. The frequency of placenta previa 90 (75\%), and accreta was 30 (25\%). Primigravida patients were 19 (15.8\%) and multigravida patients were 101 (84.1\%). hysterectomy was done in $28(23.3 \%)$ patients. Spinal anesthesia was given in $50(41 \%)$ patients while $70(58.3 \%)$ patients have general anesthesia. The spinal anesthesia was later on converted to general anesthesia in $32(26.6 \%)$ patients out of 50 (41\%). Only 12 (10\%) patients were reopened for uncontrolled bleeding and $4(3.3 \%)$ patients were sent to tertiary care hospital for further management. The general surgeon was called for help in 24 (20\%) of patients and bladder injury occurred in 25 (20.8\%) patients. No mortality was found during this period.

Conclusion: Abnormal placental placement is a threat to mother life as well fetus and team of dedicated doctor is required to deal with this emergency. Use of inotropes can help to save the precious lives.
\end{abstract}

Keywords: Abnormal placental placement, Cesarean section, General anesthesia, Placenta previa, Spinal anesthesia.

How to Cite This Article: Ahmad M. Strategies for Anesthesia Management of Cesarean Deliveries with Abnormal Placental Placement-Anesthetist's Perspective. Pak Armed Forces Med J 2021; 71(5): 1778-1781. ～doi: https://doi.org/10.51253/pafmj.v71i5.5381

This is an Open Access article distributed under the terms of the Creative Commons Attribution License (https://creativecommons.org/licenses/by-nc/4.0/), which permits unrestricted use, distribution, and reproduction in any medium, provided the original work is properly cited.

\section{INTRODUCTION}

The risk of abnormal placental placement is increased with repeated cesarean sections (c-sections), ${ }^{1}$ and different causation mechanisms are suggested for this life-threatening abnormality. The development of fibrotic adhesions and repeated visceral injury are associated with an increased rate of the abnormal placenta in otherwise healthy female.,3 The choice of anesthesia for such operations is general anesthesia but spinal/ epidural anesthesia can also be used. The issue with spinal anesthesia is difficulty in the management of uncontrolled hemorrhage and prolonged surgery in some cases. ${ }^{4}$

There are different types of abnormal placenta presentations and all contribute to major obstetric hemorrhage. Obstetric hemorrhage can be defined in four "categories": Abnormal tissue (placentation), abnormal tone (atony), abnormal coagulation, and trauma (uterine rupture, cesarean delivery). ${ }^{5}$ The abnormal

Correspondence: Dr Maqsood Ahmad, Department of Anaesthesia, Combined Military Hospital, Okara Pakistan

Received: 22 Sep 2020; revision received: 26 Apr 2021; accepted: 30 Apr 2021 placentation is a leading cause of co hysterectomies, intraoperative hemorrhage, and maternal morbidity. Placenta previa and abruptio placentae are the common cause of antepartum hemorrhage. ${ }^{6}$ Placenta accreta is the penetration of the myometrium by placental villi and the risk of accrete increases in women with Previa increases $3-61 \%$ in the quaternary C-section and it is becoming the leading cause of cesarean hysterectomy. ${ }^{7}$ The average blood loss during the delivery of patients with placenta accreta is 3-5L. ${ }^{8}$ This amount of blood loss is difficult to manage under spinal anesthesia and previously general anesthesia was advocated to manage these cases.

The choice of anesthesia for managing such cases was controversial in the past but any type of anesthesia can be used safely considering the condition of mother and fetus. The concept of "more control" during general anesthesia is not supported by literature. ${ }^{9}$ The main concerns in managing such cases are intraoperative monitoring of patients, replacement of fluid loss with crystalloid or colloids, blood, and blood products. This study aims to share the experience of managing these cases of obstetric hemorrhages during elective and 
emergency surgery with abnormal placental placement and outcome of operations.

\section{METHODOLOGY}

Total of 120 patients were included in this retrospective observational study which was started after approval of the hospital ethics committee (IERC/ ANES/14/2020), (Different hospitals for the last 7 years from 2013 to 2020). All elective, emergency, booked and unbooked cases were enrolled in this comparative study. The sample size was calculated with the WHO sample size calculator and it was 100 patients but we included 120 patients to increase the strength of the study. ${ }^{10}$ It was non probability sampling based on convenience.

Inclusion Criteria: All patients reporting for emergency or elective cesarean section with known or unknown abnormal placental placement were included.

Exclusion Criteria: Non-consenting patients were excluded.

The general anesthesia with Preoxygenation, IV propofol 120-150 mg, and suxamethonium $100 \mathrm{mg}$ were standard in all known abnormal placental placement patients followed by endo tracheal intubation (ETT) of $7 \mathrm{~mm}$. After securing ETT, the anesthesia was maintained on $100 \%$ oxygen and isoflurane mixture followed by atracurium $30 \mathrm{mg}$ in all patients. The spinal anesthesia was used in patients with low risk of bleeding and if a placental abnormality was not exactly known previously even on abdominal ultrasound. The standard 25G or 26 or 27 Quincke Babcok spinal needle was used for regional anesthesia. Each spinal anesthesia patient was co loa-ded with Ringers Lactate solution with $18 \mathrm{G}$ cannula and hyperbaric $0.5 \%$ bupivacaine $2 \mathrm{ml}$ was given at L2-3 or L3-4 space by the same anesthetist. After spinal anesthesia patients were placed supine with $10-15 \%$ position and surgery was started after the onset of sensory and motor block. The vital signs of the patients were monitored with basic vital sign monitor and the arterial line was passed in the left radial artery in cases where heavy blood loss was anticipated. We arranged 2 units of grouped and cross-matched blood for each patient known to have abnormal placental placement as a standard protocol for such patients. The records of total blood, colloids, and crystalloids transfused intra-operatively were maintained. The inotropic support with norepinephrine, adrenaline, and or ketamine was individualized for each patient. The patients either had a cesarean section followed by hysterectomy or cesarean section with preservation of the uterus. Any morbidity or mortality during surgery was also recorded.

The data thus obtained in all patients was subject to analysis with Statistical Package for the Social Sciences (SPSS) version 21. The data was presented in percentages.

\section{RESULTS}

A total of 120 patients were operated having abnormal placentation. The incidence of Placenta previa $90(75 \%)$, and Accreta was 30 (25\%). Primigravida patients were $19(15.8 \%)$ and Multigravida patients were $101(84.1 \%)$. Hysterectomy was done in $28(23.3 \%)$ patients (Table-I). Spinal anesthesia was given in $50(41 \%)$ patients while $70(58.3 \%)$ patients have general anesthesia. The spinal anesthesia was later on converted to general anesthesia in $32(26.6 \%)$ patients out of 50 (41\%). Only $12(10 \%)$ patients were reopened for uncontrolled bleeding and $4(3.3 \%)$ patients were sent to tertiary care hospital for further management (TableII). The surgeon was called for help in $24(20 \%)$ of patients and bladder injury occurred in $25(20.8 \%)$ patients. No mortality was found during this period (Table-III).

Table-I: Association of cesarean section and hysterectomy.

\begin{tabular}{l|c|c|c}
\hline \multirow{2}{*}{ Cesarean Section } & \multicolumn{2}{|c|}{ Co-Hysterectomy } & \multirow{2}{*}{$\begin{array}{c}\text { Total } \\
(\mathbf{n = 1 2 0 )}\end{array}$} \\
\cline { 2 - 3 } & Yes, $(\mathbf{n = 2 8})$ & No, $(\mathbf{n}=\mathbf{9 2})$ & \\
\hline First Operation & $3(2.5 \%)$ & $16(13.3 \%)$ & $19(15.8 \%)$ \\
\hline Second Operation & $10(8.3 \%)$ & $28(23.3 \%)$ & $38(31.6 \%)$ \\
\hline Third Operation & $9(7.5 \%)$ & $41(34 \%)$ & $50(41 \%)$ \\
\hline Fourth Operation & $6(5 \%)$ & $7(5.8 \%)$ & $13(10.8 \%)$ \\
\hline
\end{tabular}

Table-II: Frequency of placenta type in previous cesarean sections.

\begin{tabular}{l|c|c}
\hline \multirow{2}{*}{ Cesarean Section } & \multicolumn{2}{|c}{ Placenta Type } \\
\cline { 2 - 3 } & Placenta Previa & Placenta Accreta \\
\hline First Operation & $15(12.5 \%)$ & $4(3.33 \%)$ \\
\hline Second Operation & $32(26.6 \%)$ & $6(5 \%)$ \\
\hline Third Operation & $33(27.5 \%)$ & $17(14.1 \%)$ \\
\hline Fourth Operation & $10(8.3 \%)$ & $3(2.5 \%)$ \\
\hline Table-III: Percentages of operated characteristics. \\
\hline Parameter & Frequency (\%) \\
\hline Co Hysterectomy & $28(23.3 \%)$ \\
\hline Re operated & $12(10 \%)$ \\
\hline Surgeon Help & $24(20 \%)$ \\
\hline General Anesthesia & $32(26.6 \%)$ \\
\hline Spinal Anesthesia & $50(41 \%)$ \\
\hline \multicolumn{2}{l|}{ Transferred to Tertiary care } & $4(3.3 \%)$ \\
\hline
\end{tabular}

\section{DISCUSSION}

The placental abnormalities are a constant threat to the life of mother and fetus due to heavy perioperative bleeding which is very difficult to manage. ${ }^{11}$ Placenta accreta vera, placenta increta, placenta percreta, and previa are the common abnormal placental 
placements. ${ }^{12}$ There are various theories for the development of abnormal placenta but it can be found without these known causes and incidence has increased over the last several years. ${ }^{13}$

Few factors responsible for placenta previa include advanced maternal age, multiparity, history of dilation and curettage, hypertensive disorders, and smoking which are also risk factors for accreta in patients with a placenta previa. ${ }^{14}$ The placenta accreta usually resulted in cesarean hysterectomy where the placenta is left attached and hysterectomy is carried out. ${ }^{15}$ Whatsoever is the causation, the main problems are sudden intraoperative hemorrhage. To manage this massive bleeding a multidisciplinary team is mandatory to timely arrange blood and blood products, essential lifesaving drugs, and monitoring. In our hospital, we had a protocol for managing such cases in which 2 units of blood were asked for each patient with risk of bleeding and keeping up to 6 along with fresh frozen plasma and even platelets in triple 1 regime in massively bleeding patients. The arterial line was passed in the left radial artery and central venous line (CVP) was passed in high-risk cases, otherwise, two large-bore 14 or $16 \mathrm{~g}$ cannula were passed before starting anesthesia. The drugs like atropine, adrenaline, and ephedrine were diluted and given in IV boluses whereas norepine and dobutamine were given in infusions. The novel approaches to control bleeding in the placenta increta in the first trimester were uterine artery embolization as described by Majd et al. ${ }^{16}$ However this facility was not available with us. Mathew Burtelow in his article has described massive blood transfusion protocol for the management of postpartum hemorrhage and their protocols are similar to our protocols. ${ }^{17}$ The 6:4:1 ratio for blood, fresh frozen plasma, and platelets was used in their study but we followed our protocol where the ratio was 1:4 for blood and FFP's and platelets were only used if found below the normal. A study by Wang et al have mentioned the use of aortic balloon occlusion for controlling intraoperative hemorrhage in placenta previa increta and percreta but even this method is not available with us. ${ }^{18}$ Yousaf $e t$ al, has concluded in their meta-analysis about the use of endovascular interventions as an effective method of controlling hemorrhage and decreasing hysterectomy rate. ${ }^{19}$ Whatsoever is the management technique, antenatal ultrasonography helps diagnose such cases and the adopting management accordingly..$^{20}$ Similarly, intraoperative cell salvage techniques and allogenic transfusions can be very helpful in these cases as mentioned by Zeng et al..$^{21}$
There was no mortality in our patients even after transfusing 10 units of blood and blood products. The main focus was to maintain blood pressure intraoperatively and we aimed to maintain systolic blood pressure $>90 \mathrm{mmHg}$ by all means. This aim was achieved with blood loss replacement with blood and blood products and decreasing depth of anesthesia. The inotropic drugs boluses were given to support blood pressure. Thus, we have seen in our experience that if we keep the systolic blood pressure $>90 \mathrm{mmHg}$, we can hope for a better outcome. The bleeding can be so massive that despite all efforts, it becomes difficult to maintain blood pressure and sometimes ketamine IV can help in those situations. All the anesthesia gases were turned off while giving only IV ketamine $1-1.5 \mathrm{mg} / \mathrm{kg}$ body weight which being sympathomimetic temporarily supported the blood pressure.

\section{CONCLUSION}

Abnormal placental placement is a threat to mother life as well fetus and team of dedicated doctor is required to deal with this emergency. Use of inotropes can help to save the precious lives.

\section{Conflict of Interest: None.}

\section{Authors' Contribution}

MA: Direct contribution to the conception, design, analysis and interpreation of data.

\section{REFERENCES}

1. Narava S, Pokhriyal SC, Singh SB, Barpanda S, Bricker L. Outcome of multiple cesarean sections in a tertiary maternity hospital in the United Arab Emirates: A retrospective analysis. . Eur J Obstet Gynecol Reprod Bio 2020; 247(1): 143-148.

2. Hay E, Lucariello A, Contieri M, Trucillo M, Pavese L, Guerra G, et al. Differential expression of several factors involved in placental development in a normal and abnormal condition. Placenta 2020; 95(4); 1-8.

3. Turco MY, Moffett A. Development of the human placenta. Develop 2019; 15(11); 146-52.

4. Hawkins R, Evans M, Hammond S, Hartopp R, Evans E. Placenta Accreta Spectrum disorders-Peri-operative management: The role of the anesthetist. Best Pract Res Clin Obstet Gynaecol 2021; 72(1): 38-51.

5. El Gelany S, Ibrahim EM, Abdelraheim AR, Khalifa EM, Abdelhakium AK, Yousef AM, et al. Management of bleeding from morbidly adherent placenta during elective repeat cesarean section: retrospective-record-based study. BMC Pregnancy Child 2019; 19(1): 106-112.

6. Collins SL, Alemdar B, van Beekhuizen HJ, Bertholdt C, Braun T, Calda $\mathrm{P}$, et al. Evidence-based guidelines for the management of abnormally invasive placenta: recommendations from the International Society for Abnormally Invasive Placenta. Am J Obstet Gynecol 2019; 220(6): 511-526.

7. Jauniaux E, Bunce C, Grønbeck L. Prevalence, and main outcomes of placenta accreta spectrum: a systematic review and meta-analysis. Am J Obstet Gynecol 2019; 221(3): 208-218. 
8. Zhang, L, Bi S, Du L. Effect of previous placenta previa on outcome of next pregnancy: a 10-year retrospective cohort study. BMC Pregnancy Childbirth 2020; 20(1): 212-215.

9. Markley JC, Farber MK, Perlman NC, Carusi DA. Neuraxial anesthesia during cesarean delivery for placenta previa with suspected morbidly adherent placenta: a retrospective analysis. Anesth Analg 2018; 127(4): 930-938.

10. Orhan B, Everen B, Anesthesia for cesarean section in parturients with abnormal placentation: A restrospective study. Cureus 2019; 11((6): e5033.

11. Hill JR, Burghardt RC, Jones K, Long CR, Looney CR, Shin T, et al. Evidence for a placental abnormality as the major cause of mortality in first-trimester somatic cell cloned bovine fetuses. Reprod Biol 2000; 63(6): 1787-1794.

12. Rao KP, Belogolovkin V, Yankowitz J, Spinnato JA. Abnormal placentation: evidence-based diagnosis and management of placenta previa, placenta accreta, and vasa previa. Obstet Gynecol Surv 2012; 67(8): 503-519.

13. Bauer ST, Bonanno C. Abnormal placentation. In Seminars in perinatology, WB Saunders 2009; 33(2): 88-96.

14. Kollmann M, Gaulhofer J, Lang U, Klaritsch P. Placenta praevia: incidence, risk factors, and outcome. J Matern Fetal Neonatal Med 2016; 29(9): 1395-1398.
15. Higgins MF, Monteith C, Foley M. Real increasing incidence of hysterectomy for placenta accreta following pre-vious cesarean section. Eur J Obstet Gynecol Reprod Biol 2013; 171(1): 54-56.

16. Majd HS, Srikantha M, Majumdar S, Christopher B. Successful use of uterine artery embolization to treat placenta increta in the first trimester. Arch Obstet Gynaecol 2009; 279(5): 713-718.

17. Burtelow M, Riley E, Druzin M, Fontaine M, Viele M, Goodnough LT. How we treat: management of life-threatening primary postpartum hemorrhage with a standardized massive transfusion protocol. J Transfus 2007; 47(9): 1564-1572.

18. Wang YL, Su FM, Zhang HY, Wang F, Zhe RL, Shen XY. Aortic balloon occlusion for controlling intraoperative hemorrhage in patients with placenta previa increta/percreta. J Matern Fetal Neonatal Med 2017; 30(21): 2564-2568.

19. Shahin Y, Pang CL. Endovascular interventional modalities for hemorrhage control in abnormal placental implantation deliveries: a systematic review and meta-analysis. Eur Radiol 2018; 28(7): 2713-2726.

20. Silver RM. Abnormal placentation: placenta previa, vasa previa, and placenta accreta. Med J Obstet Gynecol 2015; 126(3): 654-668.

21. Zeng K, Huang W. How about "The effect of intraoperative cell salvage on allogeneic blood transfusion for patients with placenta accreta"?: an observational study. Med 2018; 97(22): e10942. 